\title{
Sexualbezogene Online-Fortbildung für Fachkräfte: Eine Einführung
}

\author{
Nicola Döringa
}

Wenn Sie als Fachkraft im Bereich Sexualmedizin, Sexualpädagogik, Sexualtherapie, Sexualberatung und in angrenzenden Feldern tätig sind und zuweilen die „Zeitschrift für Sexualforschung“ zur Hand nehmen, widmen Sie sich der informellen Fort- und Weiterbildung. Denn auch wenn die Lektüre der vorliegenden Fachzeitschrift keine formal anerkannten Fortbildungspunkte bringt, so sollte sie doch idealerweise zum Ausbau der eigenen fachlichen Kenntnisse und Fähigkeiten beitragen. Wir alle sind sehr daran gewöhnt, dass ein Großteil unserer informellen Fort- und Weiterbildungsaktivitäten über Fachliteratur läuft und damit in der Tradition der Buchdruck-Ära steht. Doch im Internet-Zeitalter gibt es inzwischen sehr viel mehr Vermittlungsformate jenseits der herkömmlichen Fachtexte. Und sie sind buchstäblich nur einen Klick entfernt.

\section{Online-Fortbildung}

Welche Online-Formate das im Einzelnen sind, und wo sich dort relevante Inhalte für Fachkräfte im Bereich Sexualität und sexuelle Gesundheit verbergen, möchte Ihnen in den folgenden Heften eine neue praxisorientierte Beitragsreihe nahebringen. Dabei geht es - wie so oft bei Online-Phänomenen - nicht um eine Verdrängung, sondern um eine sinnvolle Ergänzung und Komplementierung der bisherigen Fachliteratur. Teilweise existiert ja auch bereits eine Verschmelzung. So haben zahlreiche Thieme-Fachzeitschriften ausgewählte Artikel zu Online-Fortbildungsmodulen ausgebaut. Wer als Mediziner_in im Themengebiet Sexualität CME-Punkte sammeln will, findet auf dem Online-Fortbildungsportal des Verlags bereits Dutzende von Modulen (https://cme.thieme.de/cme-webapp/), etwa zu sexueller Sucht, sexueller Traumatisierung oder Paartherapie und Sexualität.

a Institut für Medien und Kommunikationswissenschaft, Technische Universität Ilmenau

Z Sexualforsch 2018; 31; 97-100

(c) Georg Thieme Verlag KG Stuttgart · New York

ISSN 0932-8114

DOI 10.1055/s-0044-101531 


\section{Informelle Online-Fortbildung}

Neben solchen formalen Fortbildungsangeboten bietet das Internet eine Fülle an Content für die informelle Bildung, seien es Audio- und Videobeiträge, Weblogs oder Online-Foren. Derartige sexualbezogene Online-Beiträge stammen von ausgebildeten Fachkräften aus der Wissenschaft und/ oder aus der medizinischen, psychologischen und pädagogischen Praxis, aus dem Journalismus und dem Aktivismus, und natürlich von einzelnen Betroffenen bzw. Beteiligten. Im deutsch- und englischsprachigen Raum existiert ein breites Spektrum an fortbildungsrelevanten sexualbezogenen Online-Inhalten, die es zu erschließen gilt.

\section{Beispiel: Asexualität}

Im zurückliegenden Heft hat Volkmar Sigusch (2017) in seinem Jubiläumsbeitrag die letzten 30 Jahre der „Zeitschrift für Sexualforschung“ resümiert und zeitgenössische sexuelle Trends kommentiert. Dabei betont er ausdrücklich, wie sehr es ihn freut, dass asexuelle Menschen sich heutzutage zu Wort melden, sich organisieren und öffentlich präsent sind. Tatsächlich verdankt sich unser heutiges Verständnis von Asexualität als sexueller Orientierung und Identität wesentlich der Öffentlichkeitsarbeit im Internet, durch die dann auch vermehrte Forschung möglich wurde. Wie asexuelle Menschen, die schätzungsweise 1\% der Bevölkerung darstellen, sich selbst definieren und ihre sexuellen Werdegänge beschreiben, zeigen anschaulich ihre Selbstvorstellungen in öffentlichen Online-Foren (z. B. im deutschsprachigen AVEN-Forum: https://www.aven-forum.de/).

Wer von asexuellen Menschen nicht nur lesen, sondern sie auch hören und sehen möchte, findet zahlreiche Video-Zeugnisse. Man suche auf YouTube einfach nach „I'm asexual“ oder „Ich bin asexuell“ bzw. nach den im Folgenden genannten Video-Titeln und Kanal-Namen. Fritzi beispielsweise ist eine junge Frau aus Deutschland, die sehr ausführlich über ihre asexuelle Orientierung und ihr Coming-out spricht: Erstmals äußerte sie sich im Sommer 2016 in ihrem bald 400.000 Mal abgerufenen Video „Storytime. Wie ich gemerkt habe, dass ich asexuell bin" und dann noch einmal ein Jahr später: „Ja, ich bin immer noch asexuell“. Für solche 20-minütigen Reflexionsvideos sind entsprechende Zeit und Geduld mitzubringen. Lohnenswert ist jeweils auch die Beachtung der Videokommentare, in denen sich die öffentliche Wahrnehmung von Asexualität widerspiegelt - von Anerkennung über Gleichgültigkeit bis zu Spott und Ablehnung. Amelia aus England identifiziert sich ebenfalls als asexuell und startete 2015 im Alter von 18 Jahren ihren YouTube-Kanal „AmeliaACE“ (https://www.youtube.com/ ameliaACE), auf dem sie mittlerweile über 70 Videos zum Thema Asexualität veröffentlicht hat. Mehr als 8.500 Menschen folgen bislang ihrem Kanal.

Wenn besonders pointierte Online-Videos gefragt sind - z.B. für den sexualpädagogischen Unterricht oder als Einstieg in einen Fachvortrag -, stellen diese millionenfach geklickten, vom YouTube-Kanal „Boldly“ profes- 
sionell produzierten Ein- bis Dreiminüter eine Option dar: „I'm asexual, but I am not" und „Ask an Asexual Person“.

Die Stimmen asexueller Menschen im O-Ton zu hören, ist eine sinnvolle Ergänzung zur Lektüre der Fachliteratur. Nicht zuletzt ist die AsexualityCommunity (auch: ACE Community) im Internet eine wichtige Wissensquelle und Kooperationspartnerin für die Asexualitätsforschung, wie das Team um die kanadische Psychologin und Sexualitätsforscherin Lori Brotto auf dem eigenen YouTube-Kanal erläutert („Should researchers partner with members of the asexuality community to do research?"). OnlineVideos und Online-Foren der Asexuality-Community können - bei entsprechend forschungsethischem Vorgehen - Material für inhaltsanalytische Studien liefern, und sie können dazu dienen, Teilnehmende für schriftliche und mündliche Befragungen oder Tests zu gewinnen (MacNeela und Murphy, 2015; Brotto und Yule, 2017).

\section{Praktischer Nutzen der Online-Fortbildung}

Was hier am Beispiel Asexualität skizziert wurde, gilt auch für alle möglichen anderen sexuellen Themen und Phänomene: Einschlägige OnlineQuellen wie Foren, Webvideos und Co. zu nutzen, verbessert das Verständnis. Denn hier erschließen sich - ergänzend zur Fachliteratur - vor allem die vielfältigen Stimmen der Betroffenen und Beteiligten. Zudem sind Audiobzw. Video-Fortbildungsformate für vielbeschäftigte Fachkräfte auch in solchen Situationen zu nutzen (z. B. beim Autofahren oder Kochen), in denen das Lesen nicht möglich ist. Mit einschlägigen Online-Quellen vertraut zu sein, mag sich für Sie als Fachkräfte auch deshalb als nützlich erweisen, weil Sie das Material bei Bedarf in der praktischen (z. B. beraterischen, therapeutischen, pädagogischen) Arbeit einsetzen und/oder in Forschungsprojekten darauf zurückgreifen können. Nicht zuletzt mögen einzelne Fachkräfte oder Institutionen durch gute Online-Beispiele inspiriert werden, sich zukünftig selbst mit eigenen Online-Beiträgen stärker in sozialen Medien zu positionieren.

\section{Vorschau und Einladung}

Geplant ist für die nächsten Hefte jeweils ein Beitrag zu Webvideos, zu Weblogs, zu Podcasts und zu Webinaren. Möglicherweise werden Beiträge zu Online-Foren, Online-Beratungsdiensten, Smartphone-Apps und anderen fortbildungsrelevanten Online-Formaten folgen. Dabei kann natürlich weder ein Anspruch auf Vollständigkeit noch auf umfassende Qualitätsprüfung erhoben werden. Vielmehr sollen in den bewusst kompakt gehaltenen Praxisbeiträgen jeweils exemplarisch einige einschlägige Angebote herausgegriffen werden, die der informellen sexualbezogenen Online-Fortbildung dienen mögen. Auf der Basis dieser Beispiele können bei Interesse weitere Materialien eigenständig gesammelt werden. 
Wenn Sie Fragen und Anregungen haben, die in dieser Beitragsreihe aufzugreifen sind, oder Online-Angebote kennen, die unbedingt berücksichtigt werden sollten, melden Sie sich bitte einfach bei der Autorin (Kontaktangaben siehe unten).

\section{Literatur}

Brotto L, Yule M. Asexuality: Sexual Orientation, Paraphilia, Sexual Dysfunction, or None of the Above? Arch Sex Behav 2017; 46: 619-627

MacNeela P, Murphy A. Freedom, Invisibility, and Community: A Qualitative Study of Self-Identification with Asexuality. Arch Sex Behav 2015; 44: 799-812

Sigusch V. Jubiläumsbeitrag: Minima sexualia zu 30 Jahren Zeitschrift für Sexualforschung. Z Sexualforsch 2017; 30: 379-401

Korrespondenzadresse

Prof. Dr. phil. Nicola Döring

Technische Universität Ilmenau

Institut für Medien und Kommunikationswissenschaft

Ehrenbergstr. 29 (EAZ 2217)

98693 Ilmenau

Nicola.Doering@tu-ilmenau.de 